\title{
Some results on the non-commuting graph of a finite group
}

\author{
K. Moradipour ${ }^{\mathrm{a}, *}$, Sh. Ilangovan ${ }^{\mathrm{b}}$, S. Rashid ${ }^{\mathrm{c}}$ \\ ${ }^{a}$ Department of Mathematics, Faculty of Khorramabad, Lorestan Branch, \\ Technical and Vocational University, Iran \\ b The University of Nottingham Malaysia Campus Jalan Broga, 43500 Semenyih Selangor Darul Ehsan, \\ Malaysia \\ c Department of Mathematics, College of Basic Sciences, Yadegar-e-Imam Khomeini (RAH) Branch, \\ Islamic Azad University, Tehran, Iran
}

*Corresponding author, e-mail: kayvanmrp@yahoo.com

Received 14 Apr 2019

Accepted 20 Aug 2019

\begin{abstract}
Let $G$ be a metacyclic $p$-group, and let $Z(G)$ be its center. The non-commuting graph $\Gamma_{G}$ of a metacyclic $p$ group $G$ is defined as the graph whose vertex set is $G-Z(G)$, and two distinct vertices $x$ and $y$ are connected by an edge if and only if the commutator of $x$ and $y$ is not the identity. In this paper, we give some graph theoretical properties of the non-commuting graph $\Gamma_{G}$. Particularly, we investigate planarity, completeness, clique number and chromatic number of such graph. Also, we prove that if $G_{1}$ and $G_{2}$ are isoclinic metacyclic $p$-groups, then their associated graphs are isomorphic.
\end{abstract}

KEYWORDS: Non-commuting graph, metacyclic p-group, isoclinic

\section{INTRODUCTION}

There are many ways to associate a graph to a group $^{1-4}$. In this paper, we consider the noncommuting graph of a metacyclic $p$-group $G$ which is denoted by $\Gamma_{G}$. The non-commuting graph $\Gamma_{G}$ of $G$ is defined as the graph whose vertex set, denoted by $V\left(\Gamma_{G}\right)$, is $G-Z(G)$ and the edge set $E\left(\Gamma_{G}\right)$ contains $(x, y)$ as an edge if and only if $x y \neq y x$. Note that $G$ is abelian if and only if $\Gamma_{G}$ is without vertices.

The non-commuting graph of a group $G$ was first introduced by Erdös, when he posed this problem in 1975. If $G$ is a group whose non-commuting graph has no infinite complete subgraph, is it true that there is a finite bound on the cardinalities of complete subgraphs of $G$ ? Neumann ${ }^{5}$ gave an affirmative answer to this question and this was the origin of many similar questions and research. Abdollahi et $\mathrm{al}^{6}$ studied the relation between some graph theoretical properties of $\Gamma_{G}$ and the group theory properties of $G$. A group $G$ is called metacyclic if it contains a normal cyclic subgroup whose quotient is also cyclic. Beuerle ${ }^{7}$ classified all nonabelian finite metacyclic $p$-groups which are stated in Theorems 1, 2, and 3 as follows.

Theorem 1 Let $G$ be a non-abelian metacyclic $p$ group of nilpotency class 2 . Then $G$ is isomorphic to exactly one group in the following list:

(1) $G \simeq\left\langle a, b \mid a^{p^{\alpha}}=b^{p^{\beta}}=1,[a, b]=a^{p^{\alpha-\gamma}}\right\rangle$, where $\alpha, \beta, \gamma \in \mathbb{N}, \alpha \geqslant 2 \gamma$, and $\beta \geqslant \gamma \geqslant 1$;

(2) $G \simeq Q=\left\langle a, b \mid a^{4}=1, b^{2}=[b, a]=a^{-2}\right\rangle$, the group of quaternions of order 8 .

Theorem 2 Let $p$ be an odd prime, and let $G$ be a metacyclic p-group of nilpotency class at least 3 . Then $G$ is isomorphic to exactly one group in the following list:

(3) $G \simeq\left\langle a, b \mid a^{p^{\alpha}}=b^{p^{\beta}}=1,[b, a]=a^{p^{\alpha-\gamma}}\right\rangle$, where $\alpha, \beta, \gamma \in \mathbb{N}, \gamma-1<\alpha<2 \gamma$, and $\gamma \leqslant \beta$;

(4) $G \simeq\left\langle a, b \mid a^{p^{\alpha}}=1, b^{p^{\beta}}=a^{p^{\alpha-\epsilon}},[b, a]=a^{p^{\alpha-\gamma}}\right\rangle$, where $\alpha, \beta, \gamma, \epsilon \in \mathbb{N}, \gamma-1<\alpha<2 \gamma, \gamma \leqslant \beta$, and $\alpha<\beta+\epsilon$.

Theorem 3 Let $G$ be a metacyclic 2-group of nilpotency class at least 3. If $G$ is of positive type, then $G$ is isomorphic to exactly one group in the following list:

(5) $G \simeq\left\langle a, b \mid a^{2^{\alpha}}=b^{2^{\beta}}=1,[b, a]=a^{2^{\alpha-\gamma}}\right\rangle$, where $\alpha, \beta, \gamma \in \mathbb{N}, 1+\gamma<\alpha<2 \gamma$, and $\beta \geqslant \gamma$

(6) $G \simeq\left\langle a, b \mid a^{2^{\alpha}}=1, b^{2^{\beta}}=a^{2^{\alpha-\epsilon}},[b, a]=a^{2^{\alpha-\gamma}}\right\rangle$, where $\alpha, \beta, \gamma, \epsilon \in \mathbb{N}, 1+\gamma<\alpha<2 \gamma, \gamma \leqslant \beta$, and $\alpha<\beta+\epsilon$.

If $G$ is of negative type, then $G$ is isomorphic to exactly one group in the following list:

(7) $G \simeq\left\langle a, b \mid a^{2^{\alpha}}=1, b^{2^{\beta}}=a^{2^{\alpha-1}},[b, a]=a^{-2}\right\rangle$, 
where $\alpha \geqslant 3$ and $\beta \geqslant 1$;

(8) $G \simeq\left\langle a, b \mid a^{2^{\alpha}}=b^{2^{\beta}}=1,[b, a]=a^{-2}\right\rangle$, where $\alpha \geqslant 3$ and $\beta \geqslant 1$

(9) $G \simeq\left\langle a, b \mid a^{2^{\alpha}}=b^{2^{\beta}}=1,[b, a]=a^{2^{\alpha-1}-2}\right\rangle$, where $\alpha \geqslant 3$ and $\beta \geqslant 1$;

(10) $G \simeq\langle a, b| a^{2^{\alpha}}=1, b^{2^{\beta}}=a^{2^{\alpha-1}},[b, a]=$ $\left.a^{2^{\alpha-\gamma}-2}\right\rangle$, where $\alpha-\gamma>1$ and $\beta>\gamma>1$;

(11) $G \simeq\left\langle a, b \mid a^{2^{\alpha}}=b^{2^{\beta}}=1,[b, a]=a^{2^{\alpha-\gamma}-2}\right\rangle$, where $\alpha-\gamma>1$ and $\beta \geqslant \gamma>1$.

We divide the above classification of metacyclic $p$ groups into three cases, namely, Case I, II, and III for convenience. These cases are described below. Note that for all cases due to Beuerle ${ }^{7}$ presentation, we consider $\alpha, \beta \in \mathbb{N}$ and $\epsilon, \gamma \geqslant 0$ as integers where $\gamma \leqslant \min \{\alpha-1, \beta\}$ and $\gamma+\epsilon \leqslant \alpha$. If $p=2$, then in addition $\alpha-\gamma>1$. We use the notation $[b, a]=$ $b a b^{-1} a^{-1}=a^{b} a^{-1}$ for the commutator of $b$ and a. Thus the groups of Case I have the following representation:

$$
G=\left\langle a, b: a^{p^{\alpha}}=1, b^{p^{\beta}}=a^{p^{\alpha-\epsilon}}, a^{b}=a^{r}\right\rangle,
$$

for some $\alpha, \beta, \epsilon, \gamma$, where $r=p^{\alpha-\gamma}+1$ and $p$ is an odd prime or $\alpha-\gamma \geqslant 2$. The groups of Case II are shown by

$$
G=\left\langle a, b: a^{2^{\alpha}}=1, b^{2^{\beta}}=a^{2^{\alpha-\epsilon}}, a^{b}=a^{-1}\right\rangle,
$$

where $\alpha \geqslant 2$ and $\epsilon \leqslant 1$. Finally, the groups of Case III are represented by

$$
G=\left\langle a, b: a^{2^{\alpha}}=1, b^{2^{\beta}}=a^{2^{\alpha-\epsilon}}, a^{b}=a^{r}\right\rangle,
$$

where $\alpha-\gamma \geqslant 2, \beta \geqslant \gamma>1, r=2^{\alpha-\gamma}-1$, and $\epsilon \leqslant 1$.

The groups of Cases I, II, and III cover all possible types of the classification. Indeed, they correspond to three different values of $r$ in $a^{b}=a^{r}$, or equivalently $[b, a]=a^{r-1}$ that is $r=p^{\alpha-\gamma}+1$ : $r=-1,2^{\alpha-1}-1,2^{\alpha-\gamma}-1$. Then Case I covers the types (1), (3), (4), (5), and (6), Case II covers the forms (2), (7), and (8), and Case III covers the forms (9), (10), and (11). In what follows, we will write 'metacyclic $p$-group' to mean metacyclic $p$-groups of all cases.

In this paper, we show that if $G_{1} \sim G_{2}$ is an isoclinic metacyclic $p$-group, then $\Gamma_{G_{1}} \simeq \Gamma_{G_{2}}$. We also obtain the clique number and chromatic number of the non-commuting graph $\Gamma_{G}$. In particular, we show that the graphs associated with nonisomorphic metacyclic $p$-groups in each Case I, II, and III have the same clique number and chromatic number.

\section{SOME RESULTS ON THE NON-COMMUTING GRAPHS OF METACYCLIC $p$-GROUPS AND COMPUTING $P(G)$}

This section provides a formula for the number of edges of non-commuting graph $\Gamma_{G}$ in terms of $\alpha, \beta$, and $\gamma$ mentioned in the above three representations. Some properties of $\Gamma_{G}$, and some relations between this graph and commutativity degree of $G, P(G)$, is obtained.

For a finite group $G$, let $\mathscr{A}$ denote the set of pairs of commuting elements of $G$ :

$$
\mathscr{A}=\{(a, b) \in G \times G \mid a b=b a\} .
$$

The quantity $|\mathscr{A}| /|G|^{2}$, which is denoted by $P(G)$, measures the probability of two random elements of $G$ commuting, and is called the commutativity degree of $G$. There are many results on the commutativity degree of some particular groups ${ }^{8-10}$. By Proposition 5 in Ref. 11, the number of edges in $\Gamma_{G}$, denoted by $\left|E\left(\Gamma_{G}\right)\right|$, is

$$
\left|E\left(\Gamma_{G}\right)\right|=\frac{1}{2}\left(|G|^{2}-k(G)|G|\right),
$$

where $k(G)$ is the number of conjugacy classes of group $G$. The following equation provides a formula for the commutativity degree of group $G$ in terms of the number of edges of graph $\Gamma_{G}$.

$$
P(G)=1-\frac{2\left|E\left(\Gamma_{G}\right)\right|}{|G|^{2}} .
$$

From the above consideration, we obtain the number of edges and commutativity degree of metacyclic $p$-groups in terms of $\alpha, \beta$, and $\gamma$.

For future reference, we need the following two propositions that give formulas for the order, the center, and the order of the center of metacyclic $p$ groups. For the proof, we refer to Proposition 2.5 in Ref. 7.

Proposition 1 Let $G$ be a metacyclic p-group of Case I. Then $|G|=p^{\alpha+\beta}, Z(G)=\left\langle a^{p^{\gamma}}, b^{p^{\gamma}}\right\rangle$, and $|Z(G)|=p^{\alpha+\beta-2 \gamma}$.

Proposition 2 Let $G$ be a metacyclic 2-group of Cases II and III. Then $|G|=2^{\alpha+\beta}, Z(G)=$ $\left\langle a^{2^{\alpha-1}}, b^{2^{\max \{1, \gamma\}}}\right\rangle$, and $|Z(G)|=2^{\beta-\max \{1, \gamma\}+1}$.

In the groups of Case II, since $\alpha \geqslant 3, \beta \geqslant 1$, and $\gamma \leqslant$ $\min \{\alpha-1, \beta\}$, then $\gamma \leqslant 1$. Thus $Z(G)=\left\langle a^{2^{\alpha-1}}, b^{2}\right\rangle$ and $|Z(G)|=2^{\beta}$.

In the following theorem, we obtain the number of edges of the non-commuting graph $\Gamma_{G}$ of the groups of Case I. 
Theorem 4 Let $G$ be a metacyclic p-group of Case I. Then $\left|E\left(\Gamma_{G}\right)\right|=\frac{1}{2}|G|^{2}\left(1-1 / p^{\gamma}-1 / p^{\gamma+1}+1 / p^{2 \gamma+1}\right)$.

Proof: From Theorem 4 of Ref. 12, the number of conjugacy classes of $G$ is $k(G)=|G|\left(1 / p^{\gamma}+1 / p^{\gamma+1}\right.$ $\left.1 / p^{2 \gamma+1}\right)$. Now, the result is followed by using equation (4).

The following theorem gives a formula for the number of edges in the non-commuting graph $\Gamma_{G}$ associated with the groups of Case II.

Theorem 5 Let $G$ be a metacyclic 2-group of Case II. If $a^{i} b^{j}$, where $0 \leqslant i<2^{\alpha}$ and $0 \leqslant j<2^{\beta}$, is an arbitrary element of $G$, then $\left|E\left(\Gamma_{G}\right)\right|=\frac{3}{8}|G|^{2}(1-$ $\left.1 / 2^{\alpha-1}\right)$.

Proof: Let $G \simeq\left\langle a, b \mid a^{2^{\alpha}}=b^{2^{\beta}}=1,[b, a]=a^{-2}\right\rangle$, be a split group $(\epsilon=0)$ of Case II. According to Proposition 2 we have $Z(G)=\left\langle a^{2^{\alpha-1}}, b^{2}\right\rangle$ and $|Z(G)|=2^{\beta}$. Since $G$ is a 2-generated metacyclic 2group of order $2^{\alpha+\beta}$ with the relation $b a=a^{r} b$, then each element in the group can be written uniquely in the form $a^{i} b^{j}$, where $0 \leqslant i<2^{\alpha}$ and $0 \leqslant j<2^{\beta}$. Note that in the case $[b, a]=a^{-2}$, we have $a^{b}=$ $b a b^{-1}=a^{-1}$, from which it follows that $a^{b^{j}}=a^{(-1)^{j}}$ and $a^{i b^{j}}=a^{(-1)^{j} i}$. Hence $b^{j} a^{i}=a^{i(-1)^{j}} b^{j}$. Thus it is easy to see that

$$
\begin{aligned}
& \left(a^{i} b^{j}\right)^{a^{s} b^{t}}=a^{(-1)^{t}\left((i+s)-(-1)^{j} s\right)} b^{j} \\
& = \begin{cases}a^{i(-1)^{\mathrm{t}}} b^{j}, & j \text { is even, } \\
a^{(-1)^{\mathrm{t}}(i+2 s)} b^{j}, & j \text { is odd. }\end{cases}
\end{aligned}
$$

Hence there are three cases of elements $x$ in the group $G$. First, if $x \in Z(G)$, then $x^{G}=\{x\}$ and the number of conjugacy classes of this type equals $|Z(G)|$ which is already given. Next, if $x=a^{i} b^{j}$ is non-central and $j$ is even, then $i \neq 0,2^{\alpha-1}$, so in this case $x^{G}=\left\{a^{i} b^{j}, a^{-i} b^{j}\right\}=\left\{a^{i}, a^{-i}\right\} b^{j}$ has two elements. Hence the number of conjugacy classes of such elements equals $\frac{1}{2}\left(2^{\alpha}-2\right) 2^{\beta-1}=\left(2^{\alpha-1}-1\right) 2^{\beta-1}$, where $2^{\beta-1}$ is the number of even $j$. Lastly, if $x=$ $a^{i} b^{j}$ is non-central and $j$ is odd, then

$$
\begin{aligned}
x^{G} & =\left\{a^{i+2 s} b^{j}, a^{-(i+2 s)} b^{j} \mid 0 \leqslant s<2^{\alpha}\right\} \\
& =\left\{a^{i+2 s} b^{j} \mid 0 \leqslant s<2^{\alpha}\right\}=\left\langle a^{2}\right\rangle a^{i} b^{j}
\end{aligned}
$$

has $2^{\alpha-1}$ elements and each odd $j$ contributes 2 conjugacy classes. Thus the number of conjugacy classes of such elements equals $2 \cdot 2^{\beta-1}=2^{\beta}$ where $2^{\beta-1}$ is the number of odd $j$. Hence the number of conjugacy classes of $G$ is $k(G)=2^{\beta}+\left(2^{\alpha-1}-\right.$ 1) $2^{\beta-1}+2^{\beta}=\frac{1}{4}|G|\left(1+3 / 2^{\alpha-1}\right)$. The results of the split case are used to find a similar formula for $k(G)$ in the non-split case. We observe that $k(G)$ for both cases are the same. Now, using (4), we arrive at the result.

The following theorem gives a formula for the number of edges of graph $\Gamma_{G}$ in the Case III groups.

Theorem 6 Let $\Gamma_{G}$ be the non-commuting graph of metacyclic 2-group of Case III. Then

$$
\left|E\left(\Gamma_{G}\right)\right|=\frac{1}{2}|G|^{2}\left(1-\frac{2^{\gamma}-1}{2^{2 \gamma-1}}-\frac{1}{2^{\gamma+2}}-\frac{3}{2^{\alpha+1}}\right) .
$$

Proof: The number of conjugacy classes of groups of Case III is

$$
\begin{aligned}
k(G) & =\left(2^{\gamma}-1\right) 2^{\alpha+\beta-2 \gamma-1}+2^{\alpha+\beta-\gamma-2}+3 \cdot 2^{\beta-1} \\
& =|G|\left(\frac{2^{\gamma}-1}{2^{2 \gamma-1}}+\frac{1}{2^{\gamma+2}}+\frac{3}{2^{\alpha+1}}\right),
\end{aligned}
$$

(see also Ref. 13). Now, by combining recent formula and (4), the result is obtained.

We now provide explicit formulas for the commutativity degree of the groups of Cases I, II, and III.

Corollary 1 Let $\Gamma_{G}$ be a non-commuting graph associated with the metacyclic p-groups of Case I. Then $P(G)=1 / p^{\gamma}+1 / p^{\gamma+1}-1 / p^{2 \gamma+1}$.

Proof: The proof can be deduced by Theorem 4 and equation (5).

Now, calculation of the commutativity degree for the groups of Cases II and III is a straightforward consequence of Theorems 5 and 6.

Corollary 2 Let $G$ be a group of Case II, and let $\Gamma_{G}$ be its non-commuting graph. If $a^{i} b^{j}$ is an arbitrary element of $G$, where $0 \leqslant i<2^{\alpha}, 0 \leqslant j<2^{\beta}$, then $P(G)=\frac{1}{4}\left(1+3 / 2^{\alpha-1}\right)$.

Proof: It is straightforward from Theorem 5 and (5).

Corollary 3 Consider the metacyclic 2-group $G$ of Case III. If $\Gamma_{G}$ is the non-commuting graph of $G$, then $P(G)=\left(2^{\gamma}-1\right) / 2^{2 \gamma-1}+1 / 2^{\gamma+2}+3 / 2^{\alpha+1}$.

Proof: The result is obtained directly by using Theorem 6 and (5).

Proposition 3 Let $G_{1}$ and $G_{2}$ be two metacyclic $p$ groups of Case I. If their associate graphs $\Gamma_{G_{1}}$ and $\Gamma_{G_{2}}$ are isomorphic, then $P\left(G_{1}\right)=P\left(G_{2}\right)$.

Proof: Suppose that $G_{1}$ and $G_{2}$ are two groups of Case I, using the proof of Theorem 4 in Ref. 12, we 
have $k\left(G_{1}\right)=k\left(G_{2}\right)$. Now, by applying Proposition 1 and (4), we obtain

$$
\begin{aligned}
\left|E\left(\Gamma_{G_{1}}\right)\right| & =\frac{\left|G_{1}\right|}{2}\left(\left|G_{1}\right|-k\left(G_{1}\right)\right) \\
& =\frac{\left|G_{2}\right|}{2}\left(\left|G_{2}\right|-k\left(G_{2}\right)\right)=\left|E\left(\Gamma_{G_{2}}\right)\right| .
\end{aligned}
$$

From this equality and v(5), the result follows. We use the results of Theorems 5 and 6 to pose a similar argument for proving the identity $P\left(G_{1}\right)=P\left(G_{2}\right)$, when $G_{1}$ and $G_{2}$ are the groups of Case II or III.

In the following example, we see that the noncommuting graph of metacyclic 2-group $D_{2^{\alpha+1}}$ is not complete. But, this graph is hamiltonian, and the commutativity degree $P(G)=\frac{5}{8}$, where $\alpha=2$.

Example 1 Consider the metacyclic 2-group $D_{2^{\alpha+1}}=\left\langle a, b: a^{2^{\alpha}}=b^{2}=1, a^{b}=a^{-1}\right\rangle$. The degree of every vertex of the non-commuting graph $\Gamma_{D_{2 \alpha+1}}$ can be calculated as follows: $\operatorname{deg}\left(a^{i}\right)=2^{\alpha}, i \neq 2^{\alpha-1}$; $\operatorname{deg}(b)=2^{\alpha+1}-4 ; \operatorname{deg}\left(a^{i} b\right)=2^{\alpha+1}-4, i \neq 2^{\alpha-1}$, and $\operatorname{deg}\left(a^{2^{\alpha-1}} b\right)=2^{\alpha+1}-4$. Since $\left|V\left(\Gamma_{D_{2 \alpha+1}}\right)\right|=2^{\alpha+1}-2$, $\operatorname{deg}(x)=2^{\alpha}-1$ and $\operatorname{deg}(x) \neq 2^{\alpha}-3$ for every vertex in the graph, so $\Gamma_{D_{2} \alpha+1}$ is hamiltonian and non-complete. We can extend this result to any metacyclic $p$-group.

Proposition 4 The non-commuting graph of every metacyclic p-group is not complete.

Proof: Suppose that $\Gamma_{G}$ is a complete graph. Then for each $x \in G-Z(G), \operatorname{deg}(x)=|G|-|Z(G)|-1$. On the other hand, we know that $\operatorname{deg}(x)=|G|-$ $\left|C_{G}(x)\right|$. Hence $|G|-|Z(G)|-1=|G|-\left|C_{G}(x)\right|$. Thus $\left|C_{G}(x)\right|=|Z(G)|+1$. Since $|Z(G)|$ divides $\left|C_{G}(x)\right|$, thus $|Z(G)|=1$, which is a contradiction to Propositions 1 and 2.

The above proposition shows that noncommuting complete graph associated with metacyclic p-groups cannot be constructed, or equivalently there is no metacyclic $p$-group with commutativity degree

$$
P(G)=\frac{2|Z(G)|}{|G|}-\frac{|Z(G)|^{2}}{|G|^{2}}+\frac{1}{|G|}-\frac{|Z(G)|}{|G|^{2}} .
$$

Proposition 5 The non-commuting graph of every metacyclic p-group is hamiltonian.

Proof: For non-commuting graph $\Gamma_{G}$, we have $\operatorname{deg}(x)=\left|G-C_{G}(x)\right|$, also since $x \in G-Z(G)$, $C_{G}(x) \neq G$, then $|G| /\left|C_{G}(x)\right| \neq 1$. Thus
$|G| /\left|C_{G}(x)\right| \geqslant 2$, that is $|G| \geqslant 2\left|C_{G}(x)\right|$. Hence

$$
\begin{aligned}
|G|-|Z(G)| & <|G|=2|G|-|G| \\
& \leqslant 2|G|-2\left|C_{G}(x)\right|=2 \operatorname{deg}(x) .
\end{aligned}
$$

Hence $\operatorname{deg}(x)>\frac{1}{2}(|G|-|Z(G)|)=\frac{1}{2}\left|V\left(\Gamma_{G}\right)\right|$. Also, since $G$ is non-abelian, $\left|V\left(\Gamma_{G}\right)\right| \geqslant 3$. Thus using Dirac's condition (Theorem 4.3 in Ref. 14), the result follows.

Corollary 4 Let $G$ be a metacyclic p-group. Then the non-commuting graph $\Gamma_{G}$ is connected.

Proof: This is an immediate result of the proof of Proposition 5.

We recall that the star graph $K_{1, n}$ is a tree on $n$ vertices with one vertex having degree $n-1$ and the others having degree 1.

Corollary 5 There is no metacyclic p-group $G$ with non-commuting star graph.

Proof: It is straightforward from the results of the proof of Proposition 5.

This corollary also shows that the noncommuting star graph associated with a metacyclic $p$-group cannot be constructed, and there is no metacyclic $p$-group with commutativity degree $P(G)=1-2(|G|-|Z(G)|-1) /|G|^{2}$.

In the following corollary, we prove that there is no complete bipartite non-commuting graph, or equivalently there is no metacyclic $p$-group $G$ with commutativity degree

$$
P(G)=1+\frac{|Z(G)|}{|G|}-\frac{|Z(G)|^{2}}{|G|^{2}} .
$$

Using Propositions 1 and 2, there is no metacyclic $p$-group ( $p$ is an odd prime) with commutativity degree $P(G)=1+1 / p^{2 \gamma}-1 / p^{4 \gamma}$, where $\gamma \geqslant 1$, and there is no metacyclic 2-group with commutativity degree $P(G)=1+1 / 2^{\alpha}-1 / 2^{2 \alpha}$, where $\alpha \geqslant 2$.

We recall that a complete bipartite graph is a bipartite graph with bipartition $(X, Y)$ in which each vertex of $X$ is joined to each vertex of $Y$, if $|X|=m$ and $|Y|=n$, such a graph will be denoted by $K_{m, n}$.

Corollary 6 There is no non-commuting complete bipartite graph for any metacyclic p-group $G$.

Proof: It is also a result of the proof of Proposition 5. If $\Gamma_{G}$ is a bipartite graph with bipartition $(X, Y)$ where $X \neq Y$, then $\Gamma_{G}$ is non-hamiltonian ${ }^{14}$.

Proposition 6 Consider a metacyclic p-group $G$. The graph $\Gamma_{G}$ is planar if and only if $G \simeq Q_{8}$ or $D_{8}$, the quaternion group and the dihedral group of order 8 , respectively. 
Proof: If $G$ has nilpotency class 2 then $G$ is isomorphic to $Q_{8}$. It is easy to see that $\Gamma_{Q_{8}}$ is a planar graph. Let the nilpotency class of $G$ be at least 3. Then from Theorem 3, $G$ is isomorphic to $D_{8}$, and $\Gamma_{D_{8}}$ is a planar graph. Now we suppose that $\Gamma_{G}$ is planar. Then by using Corollary 9.5.3 in Ref. 14 there exists a vertex $x \in G-Z(G)$ such that $\operatorname{deg}(x) \leqslant 5$. Hence $\operatorname{deg}(x)=|G|-\left|C_{G}(x)\right| \leqslant 5$. From which it follows that $\left|C_{G}(x)\right| \leqslant \frac{1}{2}|G|$, and so $|G| \leqslant 10$. Since $G$ is non-abelian metacyclic $p$-group of order $p^{\alpha+\beta}$, then $p^{\alpha+\beta} \leqslant 10$, where $\alpha \geqslant 2, \beta \geqslant 1$, and $p \geqslant 2$. Thus $|G|=8$. Since $G$ is metacyclic, $G$ is isomorphic to $Q_{8}$ or $D_{8}$.

Theorem 7 Let $G$ be metacyclic 2-group of Case II or III. Then $\chi\left(\Gamma_{G / N}\right) \leqslant \chi\left(\Gamma_{G}\right) \leqslant 2^{\alpha+\gamma-1}$.

Proof: Since $G$ is a metacyclic 2-group, $G$ contains a normal subgroup $K$. Suppose that $\chi\left(\Gamma_{G}\right)=m$. Then using Lemma 4.1 in Ref. 6, $m$ is the minimum number of abelian subgroups of $G$ whose union is $G$. We let $G_{1}, G_{2}, \ldots, G_{m}$ represent the abelian subgroups of $G$, so we have $G=\cup_{i=1}^{m} G_{i}$. Furthermore, since $K$ is a normal subgroup of $G$, thus $G / K=\cup_{i=1}^{m} K G_{i} / K$. On the other hand, we have $K G_{i} / K \simeq G_{i} /\left(K \cap G_{i}\right)$ which is an abelian subgroup of $G / K$. Hence the vertices of graph $\Gamma_{G / K}$ in $K G_{i} / K$ are not adjacent, from which it follows that $\chi\left(\Gamma_{G / K}\right) \leqslant m=\chi\left(\Gamma_{G}\right)$. Now using Proposition 2, we have $[G: Z(G)]=2^{\alpha+\gamma-1}$. Thus $G=\cup_{i=1}^{2^{\alpha+\gamma-1}} x_{i} Z(G)$ for $x_{i} \in G$, when $1 \leqslant i \leqslant 2^{\alpha+\gamma-1}$. It is easy to see that $x_{i} Z(G)$ is an independent subset in $\Gamma_{G}$ for each $i$. Since $G-Z(G)=\bigcup_{i=1}^{2^{\alpha+\gamma-1}} x_{i} Z(G)$, so $\chi\left(\Gamma_{G}\right) \leqslant 2^{\alpha+\gamma-1}$.

From the above consideration, we have a similar result for the metacyclic p-groups of Case I.

Theorem 8 Let $G$ be a finite metacyclic p-group of Case I. Then $\chi\left(\Gamma_{G / N}\right) \leqslant \chi\left(\Gamma_{G}\right) \leqslant p^{2 \gamma}$.

Proof: We use a method similar to that in Theorem 7 to find an upper bound for the chromatic number of graph $\Gamma_{G}$. From Proposition 1 and a straightforward calculation, we conclude that $[G: Z(G)]=p^{2 \gamma}$. Hence $\chi\left(\Gamma_{G / N}\right) \leqslant \chi\left(\Gamma_{G}\right) \leqslant p^{2 \gamma}$.

It is easy to see that if $G_{1} \simeq G_{2}$ is isomorphic metacyclic $p$-groups then $\Gamma_{G_{1}} \simeq \Gamma_{G_{1}}$, but the converse is not true. Two metacyclic 2-groups $D_{8}$ and $Q_{8}$ are not isomorphic, but their non-commuting graphs are isomorphic, that is $\Gamma_{D_{8}} \simeq \Gamma_{Q_{8}}$. In Theorem 9, we show that if $G_{1} \sim G_{2}$ is isoclinic metacyclic $p$-groups, then $\Gamma_{G_{1}} \simeq \Gamma_{G_{2}}$. We need the following definition of isoclinism between two groups.

Definition 1 Let $G$ and $H$ be two groups. A pair $(\alpha, \beta)$ is called an isoclinism from $G$ to $H$ if $\alpha$ is an isomorphism from $G / Z(G)$ to $H / Z(H), \beta$ is also an isomorphism from $G^{\prime}$ to $H^{\prime}$ and $\beta\left(\left[g_{1}, g_{2}\right]\right)=$ $\left[h_{1}, h_{2}\right]$ whenever $h_{i} \in \alpha\left(g_{i} Z(G)\right)$ for all $g_{i} \in G$ and $h_{i} \in H, 1 \leqslant i \leqslant 2$. If there is an isoclinism from $G$ to $H$, we say that $G$ and $H$ are isoclinic, written briefly as $G \sim H$.

Theorem 9 Let $G_{1} \sim G_{2}$ be isoclinic metacyclic $p$ groups. Then $\Gamma_{G_{1}} \simeq \Gamma_{G_{1}}$.

Proof: Let $G_{1}$ and $G_{2}$ be two groups of Case I. Then using Proposition $1,\left|Z\left(G_{1}\right)\right|=\left|Z\left(G_{2}\right)\right|$, and clearly $\left|V\left(\Gamma_{G_{1}}\right)\right|=\left|V\left(\Gamma_{G_{2}}\right)\right|$. Also, $\left|G_{1} / Z\left(G_{1}\right)\right|=$ $\left|G_{2} / Z\left(G_{2}\right)\right|=p^{2 \gamma}$. Assume that $\left\{g_{1}, g_{2}, \ldots, g_{p^{2 \gamma}}\right\}$ is the set of left transversal for $G_{1} / Z\left(G_{1}\right)$. Similarly, suppose that $\left\{g_{1}^{\prime}, g_{2}^{\prime}, \ldots, g_{p^{2 \gamma}}^{\prime}\right\}$ is the set of left transversal for $G_{2} / Z\left(G_{2}\right)$. Suppose that $\theta: Z\left(G_{1}\right) \rightarrow$ $Z\left(G_{2}\right)$ is a bijection which maps elements of $G_{1}$ to $G_{2}$. By isomorphism $\alpha: G_{1} / Z\left(G_{1}\right) \rightarrow G_{2} / Z\left(G_{2}\right)$ we have $g_{i} Z\left(G_{1}\right) \mapsto g_{i}^{\prime} Z\left(G_{2}\right)$ for $1 \leqslant i \leqslant p^{2 \gamma}$. In addition, the isomorphism $\beta:\left[G_{1}, G_{1}\right] \rightarrow\left[G_{2}, G_{2}\right]$ can be defined by $\beta\left(\left[g_{i} z_{1}, g_{j} z_{2}\right]\right)=\left[g_{i}^{\prime} z_{1}^{\prime}, g_{j}^{\prime} z_{2}^{\prime}\right]$, where $1 \leqslant i, j \leqslant p^{2 \gamma}, z_{1}, z_{2} \in Z\left(G_{1}\right)$ and $z_{1}^{\prime}, z_{2}^{\prime} \in Z\left(G_{2}\right)$. Now we introduce $\varphi: G_{1} \backslash Z\left(G_{1}\right) \rightarrow G_{2} \backslash Z\left(G_{2}\right)$ that maps $g_{i} z_{1} \mapsto g_{i}^{\prime} \theta\left(z_{1}\right)$ where $z_{1} \in Z\left(G_{1}\right)$. The map $\varphi$ is the favorite bijection between the set of vertices of $\Gamma_{G_{1}}$ and $\Gamma_{G_{2}}$, which clearly preserves the graphs edges.

For the groups of Cases II and III, a similar method can be applied to obtain the results of Theorem 9 .

In the following theorems we obtain the exact number of clique number and chromatic number of non-commuting graphs for each case of metacyclic $p$-groups, and we show that they are identical.

Theorem 10 Let $G$ be a metacyclic 2-group of Case II or III. Then $\omega\left(\Gamma_{G}\right)=\chi\left(\Gamma_{G}\right)$.

Proof: We only give the proof of Case III groups since the proof for the groups of Case II is similar. By Proposition 2, we have $Z(G)=\left\langle a^{2^{\alpha-1}}, b^{2^{\gamma}}\right\rangle$ and $|Z(G)|=2^{\beta-\gamma+1}$, when $\gamma>1$. Thus we have $[G$ : $Z(G)]=2^{\alpha+\gamma-1}$. Let $T=\left\{a^{i} b^{j}: 0 \leqslant i \leqslant 2^{\alpha-1}-1,0 \leqslant\right.$ $\left.j \leqslant 2^{\gamma}-1\right\}$ be a set of left transversal of $Z(G)$ in $G$, which includes $2^{\alpha-1}-1$ elements of the form $a^{i}$, $(i \neq 0)$ and $2^{\gamma}$ elements of the form $b^{j}$. Also, this set contains $\left(2^{\alpha-1}-1\right)\left(2^{\gamma}-1\right)$ elements of the form $a^{i} b^{j}$, in which $i$ and $j$ are not equal to zero at the same time. Furthermore, the vertices of $a^{i} b^{j}$ and $a^{r} b^{s}$ in the partition $G / Z(G)$, where $1 \leqslant i, r \leqslant 2^{\alpha-1}-1$ and $1 \leqslant j, s \leqslant 2^{\gamma}-1$, are adjacent. Also, the vertices of $a^{i}$ and $b^{j}$ are adjacent with $a^{i} b^{j}$. But, clearly two vertices $a^{i}$ and $a^{r}$, also the vertices $b^{j}$ and $b^{s}$, are not 
adjacent. Hence, according to the above discussion, the set:

$\mathscr{C}_{m, n}=\left\{a^{m}, b^{n}, a^{i} b^{j}: 1 \leqslant i \leqslant 2^{\alpha-1}-1,1 \leqslant j \leqslant 2^{\gamma}-1\right\}$,

in which $1 \leqslant m<2^{\alpha-1}$ and $1 \leqslant n<2^{\gamma}$, is a clique in the graph $\Gamma_{G}$ with maximum size. Hence $\left|\mathscr{C}_{m, n}\right|=$ $\left(2^{\alpha-1}-1\right)\left(2^{\gamma}-1\right)+2=\omega\left(\Gamma_{G}\right)$. Next we obtain the chromatic number of $\Gamma_{G}$. It is clear that the vertices in the set $A=\left\{a^{i}: 1 \leqslant i \leqslant 2^{\alpha-1}-1\right\}$ are not adjacent, and these vertices have the same colour. Also, the vertices $B=\left\{b^{j} \mid 1 \leqslant j \leqslant 2^{\gamma}-1\right\}$ are not adjacent and these vertices also have the same colour. Since $a^{i}$ and $b^{i}$ are adjacent, they have different colours. On the other hand, the vertices of the set $C=\left\{a^{i} b^{j}\right.$ : $\left.1 \leqslant i \leqslant 2^{\alpha-1}-1,1 \leqslant j \leqslant 2^{\gamma}-1\right\}$ are adjacent. Thus we have $\left(2^{\alpha-1}-1\right)\left(2^{\gamma}-1\right)$-colouring vertices. Using the above considerations, we have the following set:

$\mathscr{X}_{p, q}=\left\{a^{p}, b^{q}, a^{i} b^{j}: 1 \leqslant i \leqslant 2^{\alpha-1}-1,1 \leqslant j \leqslant 2^{\gamma}-1\right\}$, where $1 \leqslant p<2^{\alpha-1}$ and $1 \leqslant q<2^{\gamma}$. Hence the induced subgraph on $\mathscr{X}_{p, q}$ is a complete graph which has $\left(2^{\alpha-1}-1\right)\left(2^{\gamma}-1\right)+2=\left|\mathscr{X}_{p, q}\right|=\chi\left(\Gamma_{G}\right)$ vertices colouring with minimum size. In the case $\gamma=1$, we have $\omega\left(\Gamma_{G}\right)=\chi\left(\Gamma_{G}\right)=2^{\alpha-1}+1$, which is the clique number and chromatic number in the graph $\Gamma_{G}$ of the groups of Case II.

These results can be extended to the metacyclic $p$-groups of Case I in the following theorem.

Theorem 11 Let $G$ be a metacyclic p-group of Case I. Then $\omega\left(\Gamma_{G}\right)=\chi\left(\Gamma_{G}\right)=\left(p^{\gamma}-1\right)^{2}+2$.

Proof: Recall from Proposition 1 that $|G|=p^{\alpha+\beta}$ and $Z(G)=\left\langle a^{p^{\gamma}}, b^{p^{\gamma}}\right\rangle$, also we have $|Z(G)|=p^{\alpha+\beta-2 \gamma}$. We consider $T=\left\{a^{i} b^{j}: 0 \leqslant i, j<p^{\gamma}\right\}$ as a set of left transversal of $Z(G)$ in $G$. By using a similar method as in the proof of Theorem 10, we have $\omega\left(\Gamma_{G}\right)=$ $\left(p^{\gamma}-1\right)\left(p^{\gamma}-1\right)+2=\chi\left(\Gamma_{G}\right)$ as claimed.

\section{REFERENCES}

1. Beck I (1988) Coloring of commutative rings. J Algebra 116, 208-226.

2. Bertram EA (1983) Some applications of graph theory to finite groups. Discrete Math 44, 31-43.

3. Erfanian A, Tolue B (2012) Relative non nil- $n$ graphs of finite groups. Scienceasia 38, 201-206.

4. Segev Y (2001) The commuting graph of minimal nonsolvable groups. Geom Dedicata 88, 55-66.

5. Neumann BH (1976) A problem of Paul Erdos on groups. J Aust Math Soc Ser A 21, 467-472.

6. Abdollahi A, Akbari S, Maimani HR (2006) Noncommuting graph of a group. J Algebra 298, 468-492.

7. Beuerle JR (2005) An elementary classification of finite metacyclic $p$-groups of class at least three. Algebra Colloq 12, 553-562.

8. Erdos P, Turan P (1968) On some problems of statistical group theory. Acta Math Acad Sci Hung 19, 413-435.

9. Rezaei R, Lescot P (2007) On the relative commutativity degree of a subgroup of a finite group. Comm Algebra 35, 4183-4197.

10. Gustafson WH (1973) What is the probability that two groups elements commute? Amer Math Monthly 80, 1031-1034.

11. Moghaddamfar AR, Shi WJ, Zhou W, Zokayi AR (2005) On noncommuting graph associated with a finite group. Siberian Math J 46, 325-332.

12. Moradipour K, Sarmin NH, Erfanian A (2012) Conjugacy classes and commuting probability in finite metacyclic $p$-groups. Scienceasia 38, 113-117.

13. Moradipour K, Sarmin NH, Erfanian A (2013) Conjugacy classes and commutativity degree of metacyclic 2-groups. Comptes Ren De Aca Bul Des Sci 46, 1363-1372.

14. Bondy JA, Murty JSR (1977) Graph Theory with Applications, Elsevier Science Publishing, New York. 